\title{
MedienPädagogik
}

www. medienpaed.com

\section{Digitale Medien, soziale Benachteiligung und soziale Distinktion}

Horst Niesyto

\begin{abstract}
Der folgende Beitrag' zeigt auf, dass kulturtheoretische Ansätze zur Mediensozialisation die Relevanz unterschiedlicher sozialer Lebenslagen unterschätzten. Am Beispiel des Themas «Digital Divide» werden Forschungsbefunde auf dem Hintergrund des Spannungsfelds von sozialer Benachteiligung und sozialer Distinktion referiert und eingeordnet. Der abschliessende Teil entwickelt ein Verständnis von milieusensibler Medienkompetenzbildung, welches die Medienpraxis der Subjekte in Zusammenhang mit vorhandenen (inneren und äusseren) Ressourcen zur Lebensbewältigung betrachtet.
\end{abstract}

\section{Ausgangsüberlegungen}

Die Förderung von Kindern und Jugendlichen aus benachteiligten Sozialmilieus ist seit vielen Jahren ein wichtiger Bestandteil des Ludwigsburger Profils der Medienpädagogik. Eine der grundlegenden Intentionen ist es, lebenswelt- mit lebenslagenorientierten Überlegungen zu verbinden. Lebenswelt-Orientierung steht für eine alltagskulturelle Subjektorientierung, Lebenslagen-Orientierung steht für die Berücksichtigung struktureller sozialer und gesellschaftlicher Bedingungen, die Deutungs- und Handlungsspielräume von Akteuren unterschiedlich stark beeinflussen. So wichtig es ist, den «Eigensinn» (Bachmair 2009, S. 171) von Kindern und Jugendlichen bei der Medienaneignung zu verstehen und zu fördern, so notwendig ist es gleichzeitig, die unterschiedlichen Bedingungen und Ressourcen zu beachten, die jeweils zur Verfügung stehen. «Ressourcen`meint an dieser Stelle nicht die persönlichen, inkorporierten Fähigkeiten und Stärken (als subjektive Ressourcen), sondern die familiären, schulischen, sozialen, kulturellen und materiellen Bedingungen und Angebote, die als äussere Ressourcen für die Subjekte zugänglich sind. Die in den letzten Jahren in Deutschland verstärkt einsetzende Debatte über soziale Ungleichheiten im Bildungswesen hat die unterschiedlichen Anregungsmilieus und Ressourcen wieder mehr ins öffentliche Bewusstsein gerückt.

Der Beitrag geht auf Vorträge zurück, die der Autor auf dem 21. Kongress der DGfE (Deutsche Gesellschaft für Erziehungswissenschaft) am 19. März 2008 in Dresden (AG 47) sowie auf dem Fachkongress «Soziale Ungleichheit - Medienpädagogik - Partizipation» am 17. Oktober 2008 in Bonn hielt (Veranstalter: JFF - Institut für Medienpädagogik in Forschung und Praxis und Abteilung Medienpädagogik/PH Ludwigsburg). 
Ausgangspunkt meiner Überlegungen sind die Arbeiten des Bielefelder Wissenschaftlers Dieter Baacke, der die medienpädagogische Theorie und Forschung in den 1980er- und 1990er-Jahren massgeblich beeinflusste. Seine jugend- und kulturtheoretisch motivierten Studien folgten einem sozialökologischen Ansatz und betonten die aktive Medienaneignung sowie eine kreativ-ästhetische und handlungsorientierte Praxis der Subjekte. 1997 schrieb Baacke in seiner letzten Monografie Medienpädagogik: «Die Widersprüche der Modernisierung werden in den Subjekten ausgetragen und verarbeitet, die damit nicht gesellschaftlichen Wirkfaktoren determinierend ausgeliefert sind, sondern selbst als Produzenten und Resultat gesellschaftlichen Wandels verstanden werden müssen» (S. 26). Das Zitat verdeutlicht eine Grundposition, die seit den 1990er-Jahren in diversen Studien zur Mediennutzung empirisch belegt werden konnte: die eigenaktive Leistung von Kindern und Jugendlichen, aus Medienangeboten auswählen zu können und nicht passiv Zielscheibe medialer Reize zu sein. Dieses Verständnis von Mediennutzung grenzt sich von monokausalen Medienwirkungstheorien ab und korrespondiert mit dem Ansatz in der allgemeinen Sozialisationsforschung, dass Kinder und Jugendliche Subjekte sind, die die Realität aktiv und produktiv verarbeiten (vgl. Hurrelmann 1995). Kinder und Jugendliche wachsen heute in Medienwelten hinein und geniessen es, unabhängig von Erwachsenen Neues zu entdecken. Sie werden Mitglied in verschiedenen Medien-Communitys, bilden eigene Vorlieben aus und demonstrieren so auch ihre Zugehörigkeit zu bzw. ihre Abgrenzung von bestimmten Szenen. Gerade für Jugendliche ist die Welt der Medien ein riesiges symbolisches Reservoir für die Bewältigung altersspezifischer Entwicklungsaufgaben. Diesem Verständnis von Mediennutzung liegen kultur- und handlungstheoretische Vorstellungen zur Mediensozialisation zugrunde. Mediennutzung wird als Teil sozialen Handelns verstanden, es wird nach der Bedeutung der Medien im Alltag gefragt (u. a. Charlton/Neumann 1986; Baacke u. a. 1990).

Wenngleich diese jugendkultur- und handlungstheoretischen Ansätze wesentlich dazu beitrugen, die subjektiven Formen der Medienaneignung besser zu verstehen, so stellte z. B. Charlton 1997 selbstkritisch fest, dass der von ihm präferierte, sog. strukturanalytische Forschungsansatz auch einige ernstzunehmende Vorbehalte und kritische Anmerkungen evozierte, z. B. den Hinweis auf die zu geringe Berücksichtigung der sozialen Schicht (Mediennutzer), die mangelnde Differenzierung nach spezifischen sozialen Problemlagen, die Überschätzung der Souveränität der (kindlichen) Rezipienten gegenüber dem Medium (Charlton 1997, S. 32f.). Ähnliche kritische Hinweise müssen sich diverse Medienaneignungs-Studien gefallen lassen, die in den vergangenen 15 bis 20 Jahren im Kontext einer einseitigen Rezeption der Cultural Studies in Deutschland entstanden. Einseitig war diese Rezeption insofern, weil die entsprechenden Studien den Schwerpunkt phänomenologisch und kulturalistisch verkürzt auf die Beschreibung jugendkultureller Aneignungsformen legten und die Analyse und Relevanz struktureller Rah- 
menbedingungen für den Aneignungsprozess vernachlässigten. Die Konzentration auf die Akteursperspektive, das Postulat des sautonomen Subjekts` und die damit verbundenen Attribute (Selbststeuerung, Selbstverortung, Wahlfreiheit etc.) sind aus subjekt- und kulturtheoretischer Perspektive zwar nachvollziehbar, entkoppelt jedoch diese Perspektive zu sehr von unterschiedlichen lebenslagenbezogenen Ressourcen und 〈Kapitalsorten〉 (Bourdieu 1970) und unterschätzt in der Folge die Bedeutung dieser Faktoren für den medialen Sozialisationsprozess.

\section{Soziale Benachteiligung, Habitus und soziale Distinktion}

Aktuelle Beiträge über soziale Ungleichheit und Benachteiligung (u.a. Rehberg 2006) weisen darauf hin, dass jenseits einer zu konstatierenden Pluralisierung von Lebensstilen unterschiedliche soziale Lebenslagen und Milieus nach wie vor eine wichtige Bedeutung für die Bildungs- und Entwicklungschancen von Menschen haben. Auf dem Hintergrund empirischer Befunde der PISA-Studien (PISA-Konsortium 2004), die Zusammenhänge zwischen sozialer Herkunft und Bildungschancen belegten, entwickelte sich in den letzten Jahren eine grössere Bereitschaft, Fragen der sozialen Ungleichheit und Benachteiligung zu thematisieren.

Die Themen «soziale Benachteiligungs und ssoziale Ungleichheit) gehören in der Soziologie zu den Kernbereichen soziologischer Forschung und Theoriebildung. Der Soziologe Stefan Hradil versteht unter sozialer Ungleichheit unterschiedliche Teilhabemöglichkeiten von Personen und Personengruppen an wichtigen materiellen und immateriellen Ressourcen, z. B. Besitz oder Einkommen, höhere Bildung oder hohes Ansehen/Prestige (Hradil 2001, S. 29 f.). Soziale Ungleichheit beschreibt nach Hradil allgemein eine relative Besser- oder Schlechterstellung von Menschen im gesellschaftlichen Lebenszusammenhang, die mit dauerhaften (vorteilhaften oder nachteilhaften) Lebensbedingungen in gesellschaftlichen Beziehungsgefügen verbunden ist (ebd.). Dauerhafte nachteilhafte Lebensbedingungen führen demnach zu sozialer Benachteiligung und zur eingeschränkten Teilhabe an der Gesellschaft in verschiedenen Lebensbereichen.

In den vergangenen Jahren wurden diverse Analysen zur Bildungsbenachteiligung in Deutschland erstellt. Dem letzten Armuts- und Reichtumsbericht der Bundesregierung (2008) sind in diesem Zusammenhang u. a. folgende Daten zu entnehmen:

- Betrachtet man die berufliche Ausbildung bei Personen im Alter von 35 Jahren als abgeschlossen, so bleiben 15 Prozent der Bevölkerung dauerhaft ohne abgeschlossene berufliche Ausbildung und haben damit stark eingeschränkte Integrationschancen auf dem Arbeitsmarkt.

- Zwischen dem Bildungserfolg der Eltern und der Kinder besteht ein deutlicher Zusammenhang, der sich in der unterschiedlichen Bildungsbeteiligung der Kinder von Akademikern und Nichtakademikern zeigt. 
- 83 Prozent der Kinder von Vätern mit Hochschulabschluss studieren ebenfalls, während dies nur für 23 Prozent der Kinder von Nichtakademikern zutrifft. Besonders ausgeprägt ist dieser Zusammenhang bei der Bevölkerungsgruppe der Menschen mit Migrationshintergrund (Bundesregierung 2008, S. XIX).

Diese Daten verweisen auf das gravierende Problem der Bildungsungerechtigkeit in Deutschland. Der Bildungsforscher Jürgen Baumert spricht in Zusammenhang mit dem deutschen Schulsystem von einer (Klassengesellschaft): "Die Aufteilung in Klassen ist in Deutschland in der Schule sichtbarer als in anderen Ländern, weil mit den verschiedenen Schulformen soziale Unterschiede institutionalisiert werden, die grösser sind als die Unterschiede zwischen Wohngebieten in den USA» (DIE ZEIT vom 18.9.08, S. 87). Gleichzeitig betont Baumert, dass soziale Unterschiede in keinem Land der Welt völlig eingeebnet werden können. Die Frage sei vielmehr, wie viel Ungleichheit eine Gesellschaft sich leisten will und ertragen könne.

Mit der Tatsache der Bildungsungerechtigkeit sind in sogenannten Risikomilieus weitere Faktoren verknüpft, die strukturelle Benachteiligungen verschärfen. So formulierte beispielsweise der 11. Jugendbericht bereits 2002: «Die sozioökonomische Situation von Kindern und Jugendlichen wird nicht nur durch ihre Versorgung mit existenziellen Gütern bestimmt. Zweifelsohne sind Mangelernährung, problematische Gesundheitszustände, schlechte Versorgung mit Wohnraum, Aufwachsen in so genannten «belasteten Stadtteilen` oder unzureichende Kleidung nach wie vor wichtige Indikatoren für Problemlagen» (Bundesministerium für Familie, Senioren, Frauen und Jugend 2002, S. 144). Es geht nicht allein um Formen soziokultureller Benachteiligung, sondern um die Häufung sozioökonomischer und soziokultureller Problemlagen.

Dabei ist immer wieder darauf hinzuweisen, dass Benachteiligung als strukturelle Kategorie auf bestimmte Lebenslagen verweist, die Prozesse der Ausgrenzung befördern. Sozial und bildungsmässig benachteiligte Gruppen sind nicht homogen. Sozialgruppen können stigmatisiert werden, wenn übersehen wird, dass stets individuell unterschiedliche Verarbeitungsweisen von Benachteiligung möglich sind. Differenztheoretische Analysen unterscheiden in diesem Zusammenhang horizontale, vertikale, funktionale und soziale Differenzierungen. Diese Analysen beanspruchen eine "genauere Erfassung der unterschiedlichen Kontexte und Lebensbereiche» (Schwinn 2006, S. 1295). Differenztheoretische Ansätze setzen jedoch nicht die grundlegenden Analysen zur sozialen Ungleichheit und Benachteiligung ausser Kraft. Vielmehr geht es darum, ein tieferes Verständnis von der komplexen Wechselwirkung zwischen gesellschaftlichen Rahmenbedingungen und individueller Lebensbewältigung zu erhalten.

Der französische Soziologe Pierre Bourdieu setzte sich intensiv mit Formen sozialer Ungleichheit und ihren Entstehungsbedingungen auseinander. Mit seiner Theorie vom Habitus (Bourdieu 1970) überwand er einen Mikro-Makro-Dualismus und 
fragte nach der «Dialektik von objektiven und einverleibten Strukturen» (Bourdieu 1979, S. 164 ff.). Objektive Strukturen sind für Bourdieu nicht an sich vorhanden; es sind stets die Individuen, die als Akteurinnen und Akteure in ihren jeweiligen (sozialen) Feldern handeln. Mit Habitus ist ein System dauerhafter und übertragbarer Dispositionen zu praktischem Handeln gemeint. Diese Dispositionen sind als individuell übergreifende gesellschaftliche Muster der Wahrnehmung, des Denkens und des Handelns zu verstehen. Diese Denk- und Handlungsschemata sind weder angeboren noch determiniert, sondern gesellschaftlich erlernte Muster, die durch mehrfaches Wiederholen und durch Beobachtungen im sozialen Umfeld zu Habitualisierungen führen. Habitualisierungen haben ein Beharrungsvermögen, sind aber über reflexive Prozesse veränderbar (vgl. Krais/Gebauer 2002).

In seinem Hauptwerk Die feinen Unterschiede (Bourdieu 19871979) differenziert Bourdieu verschiedene Geschmacksdimensionen im Alltagsleben der Menschen, die mit spezifischen Habitusformen, der Zugehörigkeit zu bestimmten sozialen Räumen und der jeweiligen sozialen Herkunft verknüpft sind. Sein hauptsächliches Anliegen ist es, «die auf Immanuel Kant (1724-1804) zurückgehende bürgerliche Ideologie zu entlarven, wonach es aufgeklärten, wahren oder ästhetischen Geschmack auf der einen Seite und einen minderwertigen, «barbarischen` Geschmack auf der anderen Seite gebe» (Treibel 2004, S. 233). Bourdieu weist darauf hin, dass mit den Geschmacksunterschieden zugleich soziale Distinktionen verbunden sind. Dies bedeutet, dass der jeweils eigene Geschmack in der gesellschaftlichen Praxis dazu eingesetzt wird, um sowohl Unterschiede (gegenüber anderen Sozialgruppen) als auch Gemeinsames (gegenüber dem eigenen Milieu) zu markieren und dies im Verhalten deutlich zu machen.

Distinktionen - das hat u. a. Mikos (2007, S. 51 ff.) am Beispiel von Fernsehangeboten herausgearbeitet - dienen dazu, durch Mediennutzung und Mediendiskurse Distinktionsgewinne zu erzielen: «In der Tradition der Aufklärung kann für die herrschenden bürgerlichen Eliten nur Information bzw. Bildung das Mass aller Dinge sein, das Fernsehen als Unterhaltungsmedium unterläuft diesen Anspruch, weil die populären und ästhetischen Vergnügungen der Massen sich dem rationalen Diskurs widersetzen» (ebd., S. 55). Mikos weist zugleich darauf hin, dass es in den Gesellschaften der reflexiven Moderne einerseits zwar Homologien zwischen der Lebensweise in bestimmten Szenen und Milieus und dem Medienkonsum gebe; andererseits sei es aber auch möglich, dass Medienwissen zu einem bestimmten Medienprodukt in je verschiedenen Szenen und Milieus als Teil kulturellen bzw. symbolischen Kapitals unterschiedlich genutzt werden könne.

Inwieweit sind nun Formen von Benachteiligungen auch im Bereich der Mediennutzung zu beobachten? Inwiefern handelt es sich dabei nicht um Benachteiligung, sondern zunächst einmal um soziokulturelle Unterschiede bzw. um Formen sozialer bzw. medialer Distinktion? Im Folgenden konzentriere ich mich auf das 
Themenfeld Digital Divide, das in den vergangenen zehn Jahren an Bedeutung gewann.

\section{Mediennutzung und Digital Divide}

\subsection{Zugang zu digitalen Medien}

Die Debatte um Digital Divide bezog sich zu Beginn, also in den 1990er-Jahren, primär auf die Frage des Zugangs zu den digitalen Informationstechnologien. Drastisch zeigt sich der digitale Graben immer noch, wenn man auf die weltweite Situation blickt. So verfügt nicht einmal ein Prozent der Menschen in Afrika derzeit über einen Breitband-Internetanschluss, 70 Prozent des afrikanischen IP-Verkehrs wird kostspielig über andere Erdteile geroutet. ${ }^{2}$ Auch wenn in der sog. Dritten Welt diverse Anstrengungen gemacht werden, um die Zugänge zu digitalen Medien für mehr Menschen zu ermöglichen, muss man weiterhin von einer erheblichen digitalen Spaltung sprechen. Diese bezieht sich sowohl auf das Verhältnis zur entwickelten Welt, aber gleichzeitig auch auf die interne Kluft in diesen Ländern zwischen den Eliten, die über Zugänge verfügen, und der grossen Zahl jener, die zu den «Have-nots» gehören (vgl. Chinn/Fairlie 2006).

An dieser Stelle soll auch auf die Gruppe von Menschen hingewiesen werden, die mit Behinderungen leben. In nahezu allen medienpädagogischen Publikationen wird bislang auf ihre Situation nur randständig eingegangen. So erbrachte die europäische Studie MeAC - Measuring Progress of eAccessibility in Europe (Empirica 2007), dass es bereits beim Zugang zu digitalen Medien deutliche Benachteiligungen gibt: «People with disabilities in Europe continue to be confronted with many barriers to usage of the everyday ICT products and services that are now essential elements of social and economic life. Such eAccessibility deficits can be found across the spectrum of ICT products and services, for example telephony, TV, web and self-service terminals» (Summary der Studie). Während sich laut Studie in den USA, Kanada und Australien die Zugangsmöglichkeiten zu digitalen Medien für Menschen mit Behinderungen deutlich besser gestalten, ist in fast allen europäischen Ländern die Situation defizitär.

Heinz Moser wertete 2008 einige internationale Studien zum Thema Digital Divide aus. Er verwies u. a. auf Analysen von Holloway/Valentine, die z. B. für Grossbritannien feststellten, dass im Jahr 2000 nur 27\% der Haushalte mit schlecht ausgebildeten Personen Zugang zum Internet hatten - verglichen mit 78\% der gut Ausgebildeten (Holloway/Valentine 2003, S. 23). Ähnlich kamen Livingstone/Bober - ebenfalls für Grossbritannien - zum Schluss, dass der sozio-ökonomische Status sich stark zugunsten der Mittelklasse auswirke. Dies beträfe insbesondere auch die Breitband-Verbindungen zum Internet (vgl. Livingstone/Bober 2004, S. 12 f.). Diese und andere Analysen führten - auch in Deutschland - zu der Konsequenz,

2 Internetportal Heise vom 29.10.07: www.heise.de/newsticker/Weniger-als-vier-Prozent-der-Afrikaner-sind-online--/meldung/98115 
dass man sich durch Computerausstattungen insbesondere an Schulen, aber auch in anderen Feldern erhoffte, Menschen aus sozial und bildungsmässig benachteiligten Milieus besser zu erreichen, um dadurch ihre Partizipationschancen an den digitalen Technologien zu erhöhen.

Bezüglich des Zugangs zu digitalen Medien zeichnet sich in den letzten Jahren eine enorme Veralltäglichung insbesondere bei Jugendlichen ab (vgl. Medienpädagogischer Forschungsverbund Südwest 2008). Trotz einer tendenziellen Angleichung beim Gerätebesitz und beim Zugang zu digitalen Medien bei Jugendlichen mit unterschiedlichem formalem Bildungshintergrund ist nicht zu übersehen, dass auch in Zugangs- und Ausstattungsfragen nach wie vor Unterschiede bestehen. Es war der Medienpädagogischer Forschungsverbund selbst, der in der JIM-Studie 2005 entsprechende Daten erhob und eine schlechtere Ausstattung und schlechtere Zugänge an Grundschulen und an Hauptschulen sowie deutlich geringere Erfahrungen mit medialen Eigenproduktionen bei Hauptschülern feststellte (Medienpädagogischer Forschungsverbund 2005, S. 57). Neuere Offline-/Online-Studien verweisen ebenfalls auf soziokulturelle und bildungsbezogene Unterschiede. So stellte ein Teilbericht der ARD/ZDF-Online-Studie 2007 fest, dass «immer noch 56 Prozent aller Personen mit Volksschul- bzw. Hauptschulabschluss offline [sind], bei denen mit Abitur sind es nur 10 Prozent» (Gerhards/Mende 2007, S. 380). Die ARD/ZDF-Online-Studie 2008 sowie internationale Studien betonen vor allem die medienkulturelle Kluft zwischen den Generationen: Altersbedingte Nutzungsunterschiede seien derzeit von grösster Bedeutung (u. a. Eimeren/Frees 2008, S. 344). Die ARD/ZDF-Online-Studie 2008 präzisiert allerdings in einem Beitrag von Gerhards/Mende, dass neben den ab 60-Jährigen die Gruppe der Nicht-Berufstätigen und die Gruppe der formal niedriger Gebildeten die grössten Offliner-Gruppen ausmachen. Zu der Gruppe der formal niedriger Gebildeten zählt die Studie immerhin 14,84 Millionen Menschen in Deutschland. Die Studie stellt fest, dass die grossen Gruppen der Nicht-Berufstätigen und der formal niedriger Gebildeten «voraussichtlich auch in den kommenden Jahren in übergrosser Mehrheit «offline» bleiben werden» (Gerhards/Mende 2008, S. 365).

Bezüglich des Zugangs zu digitalen Medien lässt sich zusammenfassend festhalten, dass es weltweit gesehen nach wie vor grosse Ungleichheiten beim Zugang zu digitalen Medien gibt. Für bestimmte Gruppen wie z. B. Menschen mit Behinderungen sind nach wie vor ungleiche Zugangsbedingungen vorhanden. Bei Jugendlichen mit formal niedriger Bildung haben sich die Zugänge zu digitalen Medien im Laufe der vergangenen 10 Jahre verbessert. Hier ist künftig zu beobachten, inwieweit innerhalb der Gruppe der formal niedriger Gebildeten generationenbezogene Unterschiede in der Nutzung digitaler Medien zunehmen. Im Fokus der Aufmerksamkeit sollten weiterhin jene Teilgruppen bleiben, deren Lebenssituation durch eine Häufung von Problemlagen gekennzeichnet ist. Hier geht es nicht nur um Zugangsfragen, sondern vor allem um Fragen der Bedeutung 
und der konkreten Nutzung digitaler Medien auf dem Hintergrund der jeweiligen Lebenssituation.

\subsection{Second Digital Divide}

Mit Blick auf die Web-2.0-Nutzung analysiert Heinz Moser in Anlehnung an Hargittai (2007) einen Second Digital Divide: «Nicht der Zugang ist ungleich verteilt, sondern Unterschiede in der Qualität und Intensität der Nutzung» (Moser 2008). Das Interesse konzentriert nicht auf Zugangsfragen, sondern auf die Analyse unterschiedlicher Nutzungsbedürfnisse, Aneignungsweisen und Verwendungszwecke. Hierzu entstanden in den letzten Jahren auch im deutschsprachigen Raum mehrere Studien, die Prozesse, Formen und Funktionen der Aneignung digitaler Medien erforschten. Dabei wurden soziokulturelle Unterschiede deutlich. Diese Unterschiede beziehen sich u.a. auf folgende Punkte: Unterschiede bei den Nutzungspräferenzen und der Navigationspraxis; Unterschiede bei vorhandenen Lese-, Schreib- und (audio)visuellen Kompetenzen; Unterschiede bei der Nutzung von mehr informations- und unterhaltungsorientierten Angeboten.

So betonte die Bielefelder Studie über Digitale Ungleichheit und formalen Bildungshintergrund die Notwendigkeit, stärker auf den lebensweltlichen, soziokulturellen Hintergrund der Jugendlichen zu achten. Als ein zentraler Befund wurde festgehalten, dass die Möglichkeiten der Internetnutzung stark mit den Ausgangsbedingungen der Nutzer/innen und deren sozialem Kontext im 〈real life〉 zusammenhängen. Für die Nutzung, Beteiligung und Bildungsprozesse sei es wichtig, die unterschiedlichen Ressourcen zu berücksichtigen, die den jeweiligen jugendlichen Nutzern zur Verfügung stehen. Die Forschungsgruppe empfahl Interventionen sowohl innerhalb des Internet (zielgruppenbezogene Angebotsgestaltung) als auch ausserhalb des Internet (fähigkeitserweiternde Arbeit mit Jugendlichen in Jugendhilfe und Medienarbeit; vgl. Iske/Klein/Kutscher/Otto 2007, S. 88 f.).

Dem Medienkonvergenz Monitoring Report 2009 (Schorb u. a.) ist zu entnehmen, dass die Nutzung von Video-Plattformen mit dem Bildungshintergrund korreliert: «Die formal niedriger gebildeten, die männlichen und die jüngeren Befragten schauen sich genauso häufig wie andere Jugendliche Videos an, greifen jedoch breiter auf die verschiedenen weiteren Tätigkeitsoptionen der Videoplattformen zu. Sie nutzen die Möglichkeiten, Videos zu bewerten oder zu kommentieren, andere Nutzer auf bestimmte Videos hinzuweisen, aber auch selbst Videos einzustellen, eigene Profile zu gestalten und sich mit anderen zu vernetzen, deutlich umfassender» (ebd., S. 35). Diese Medienpraxis lasse aber - so die Autoren/-innen - «keine Rückschlüsse auf die Qualität der Aneignung von Videoplattformen im Allgemeinen sowie auf förderliche oder problematische Aspekte von Tätigkeitsoptionen im Konkreten» zu (ebd.). Des Weiteren analysiert der Report, dass es verstärkt die jüngeren, männlichen und formal niedriger gebildeten Befragten seien, «die häufiger auf die YouTube-Alternativen MyVideo, Clipfish und Sevenload zu- 
greifen. Der Grunde hierfür liege vor allem in «ihrer grösseren Affinität zu lustigen, vor allem privaten Videos und ihrer Bevorzugung von deutschsprachigen Videos» (Schorb u. a. 2009, S. 2). Der Report betrachtet dieses Wahlverhalten auch in Zusammenhang mit der starken Nutzung privater TV-Programme in Deutschland. Ein weiterer Befund verdeutlicht, dass jüngere, männliche und formal niedriger gebildet Befragte eine höhere Motivation zum Einstellen von Inhalten auf Videoplattformen haben. Als Gründe werden u. a. eine höhere Affinität gegenüber «witzigen, nutzergenerierten Inhalten» angeführt (ebd., S. 22). Daten, die aus dem qualitativen Teil des Reports gewonnen wurden, geben laut der Studie Hinweise darauf, dass es "eher die Jugendlichen mit formal höherem Bildungshintergrund sind, die dem Einstellen von privaten Inhalten ins Internet etwas kritischer gegenüberstehen oder dies als «sinnlos» betrachten» (ebd.).

Eisemann (2008) verweist in einem Überblicksbeitrag zur Nutzung von Videoplattformen durch Jugendliche auf eine amerikanische Studie des PEW Internet \& American Life Project. Diese Studie (Rainie 2008) zeige, dass weisse Amerikaner erst seit kürzerer Zeit mit der Nutzung von Videoseiten aufholen, während diese Inhalte von englischsprachigen Latinos und African Americans bereits früher stärker genutzt wurden.

In beiden Studien werden soziokulturelle Unterschiede deutlich, die nicht auf soziale Benachteiligung von Menschen mit formal niedriger Bildung hinweisen. Vielmehr zeigen die Befunde, dass Videoplattformen in besonderer Weise Jugendliche mit geringerer formaler Bildung ansprechen. Ein entscheidender Grund scheint in der Möglichkeit zur (rezeptiven wie aktiv-produktiven) Nutzung präsentativ-symbolischer Materialen (Bilder, Musik) in Verbindung mit bestimmten Themenpräferenzen zu liegen. Diese Potenziale für Selbstausdruck, Information und Kommunikation dürfen aber nicht über die Risiken hinwegtäuschen, auf die der Monitoring Report 2009 auch hinweist. Zu nennen sind vor allem die «Gefahr sich in der Angebotsfülle zu «verlieren»» (Schorb u. a. 2009, S. 38) sowie «gewalthaltige, derb-zynische oder pornographische Inhalte», die für Jugendliche zum Problem werden können, wenn sie diese Inhalte «nicht verarbeiten können oder gar als Vorbilder annehmen» ebd.).

Die familiären Milieus und das dort vorhandene Bildungskapital prägen besonders unterschiedliche Formen der Medienaneignung bei Kindern und Jugendlichen. Darauf hat u. a. Ingrid Paus-Hasebrink in Zusammenhang mit mediensozialisationstheoretischen Überlegungen und eigenen Medienstudien hingewiesen (Paus-Hasebrink 2006). Ulrike Wagner und Susanne Eggert fassten in Auswertung einer Medienkonvergenz-Studie des JFF die Differenz hinsichtlich des Mediengebrauchs von Jugendlichen mit hohem und niedrigem Bildungshintergrund wie folgt zusammen: "Jugendliche, die in bildungsbevorzugten Milieus aufwachsen und selbst höhere Bildungswege einschlagen, pflegen in der Regel einen vielfältigen und kritisch reflektierten Medienumgang. Sie nehmen insbesondere multi- 
funktionale Medien in vielfältiger Weise in Gebrauch: Neben der rezeptions- und spielorientierten Nutzung schätzen sie Computer und Internet auch als potenzielle Wissens- und Informationsquellen. Sie ziehen Medien heran, um eigene Produkte zu gestalten und um ihre sozialen Beziehungen zu pflegen und zu erweitern. Jugendliche, die in bildungsbenachteiligten Milieus aufwachsen und denen selbst keine höheren Bildungswege eröffnet werden, zeigen häufiger einen primär rezeptions- und konsumorientierten Medienumgang, der zudem oft an einseitigen Inhalten (etwa Actionangeboten) ausgerichtet ist. Sie sind weniger in der Lage, die multifunktionalen Medien als Informations- und Wissenslieferanten heranzuziehen und haben weniger Chancen, die Möglichkeiten zur Kommunikation und Partizipation auszuschöpfen und in ihr alltägliches Medienhandeln zu integrieren» (Wagner \& Eggert 2007, S. 19f.).

Dieses Summary fasst empirische Befunde zusammen, die auf Problemlagen verweisen. So ist seit langer Zeit bekannt - und dies bestätigt die Münchner Studie - dass es bezüglich vorhandener Lese- und Schreibkompetenzen deutliche Unterschiede zwischen verschiedenen Bildungs- und Sozialmilieus gibt (vgl. Groeben \& Hurrelmann 2002). Diese Unterschiede werden auch bei der Aneignung digitaler Medienangebote sichtbar. Gleichzeitig ist zu hinterfragen, welche (expliziten und impliziten) Kriterien in die Bewertung der jeweiligen Medienaneignung einfliessen. So gibt es z. B. milieuspezifische Informations- und Wissensquellen mit jeweiligen Inhalten und Darstellungsformen. Dies anzuerkennen bedeutet nicht, problematische Medienangebote und Mediennutzungsweisen gering zu schätzen oder zu übergehen. Insbesondere Jugendliche, deren familiäre Verhältnisse schwierig sind, deren Eltern sich wenig um die Entwicklung ihrer Kinder kümmern, bedürfen der Unterstützung bei ihrer Lebensbewältigung.

Eine weitere Münchner JFF-Studie über Medienaneignung in Hauptschulmilieus (Wagner 2008) konzentrierte sich auf Hauptschüler/innen und nahm gegenüber der vorigen Studie eine andere Forschungsperspektive ein. Die Studie folgte einem Ansatz, der den Blick stärker auf die persönlichen Ressourcen der Subjekte lenkte, um «ihre Fähigkeiten und Kompetenzbereiche zu entdecken, die abseits formaler Qualifikationen liegen» (Wagner 2008, S. 23). Die Befunde der Studie zeigten auf, wie Hauptschülerinnen und Hauptschüler virtuelle Welten und Web 2.0 nutzen. Deutlich wurden sowohl die Stärken in vorhandenen Wissensbeständen und Kompetenzen, aber auch die Problem- und Risikobereiche. Die Studie arbeitete heraus was es heisst - Medienhandeln ist soziales Handeln: Das soziale Umfeld ist der Hauptbezugspunkt für das Medienhandeln; soziale Beziehungen werden von den Jugendlichen mittels Medien gestaltet und organisiert; präsentativ-symbolische Ausdrucksformen (vor allem Bilder, Musik) sind Mittel und Ankerpunkt für die Artikulation und Darstellung eigener Bedürfnisse und Themen. Problem- und Risikobereiche wurden am deutlichsten im Bereich der Datenweitergabe beobachtet, 
z. B. in Zusammenhang mit der Weitergabe des Passwortes als eine Art «Vertrauensbeweis> zwischen Freunden (Wagner 2008, S. 204).

Diese Befunde verweisen auf eine grosse Schnittmenge mit Ergebnissen aus Projekten medienpädagogischer Praxisforschung, die den letzten Jahren an der Pädagogischen Hochschule Ludwigsburg durchgeführt wurden. Dies betrifft insbesondere den Aspekt «präsentativ-symbolische Ausdrucksformen`: die grosse Bedeutung von Bildern, Musik und Körpersprache bei Selbstausdruck und Kommunikation mit Medien (Niesyto 2003, Witzke 2004, Maurer 2004, Holzwarth 2008). Auch die Verknüpfung von Medienerfahrungen mit dem eigenen sozialen Umfeld deckt sich mit Forschungsbefunden aus den Projekten in Ludwigsburg. Diese Verknüpfungen lassen sich theoretisch auch als «jugendkulturelle Symbolmilieus» fassen: Verbindungen von sozialen Settings der Lebensbewältigung und medienbezogenen Symbolmustern (als medialen Settings; vgl. Niesyto 2002). Die Integration unterschiedlicher symbolischer Ausdrucksformen, ein «Medienspiel) mit Angeboten, die Möglichkeiten zu Identifikationen, zu Grenzüberschreitungen und zum Ausprobieren jugendlicher Phantasien - diese medienästhetischen Erfahrungspotenziale sind für Jugendliche besonders attraktiv. Diese u. a. Potenziale digitaler Medien werden von den Jugendlichen unterschiedlich genutzt. Ihre jeweilige Mediensozialisation findet im Spannungsfeld von unterschiedlichen sozialen Lebenslagen, dem gesellschaftlichen Medienangebot, altersbezogenen Entwicklungsaufgaben und individuell-biografischen Orientierungen statt. Die Förderung von Medienkompetenzen bei Jugendlichen muss bei der Analyse der jeweiligen Mediensozialisation ansetzen und unterschiedliche soziale Lebenslagen (neben anderen wichtigen Faktoren wie Geschlecht, Alter, ethnische Herkunft) berücksichtigen. ${ }^{3}$ Theorien zur Mediensozialisation und zur Medienkompetenzbildung sind deshalb (auch) milieusensibel zu entwickeln.

\section{$4 \quad$ Medienkompetenzbildung und soziokulturelle Unterschiede}

Zur Bestimmung von Medienkompetenz gab es bislang vor allem zwei Zugangsweisen:

- Der Zugang über die individuelle Mediennutzung und Medienaneignung und damit verbundener Selbsteinschätzungen zur Medienkompetenz;

- Der Zugang über gesellschaftliche Anforderungen und damit verbundener Medienkompetenz-Definitionen und -Standards.

Die These ist, dass in der Vergangenheit jeweils eine Seite zu stark betont wurde: entweder Vereinseitigungen in Richtung subjekttheoretischer Auffassungen (z. B.

3 Die von Treumann u. a. (2007) herausgegebene Forschungsstudie «Medienhandeln Jugendlicher» ermittelte mit Hilfe clusteranalytischer Verfahren sieben Typen jugendlichen Medienhandelns. Eine sekundäranalytische Auswertung dieser breit angelegten, empirischen Medienstudie unter Aspekten sozialer Ungleichheit wäre wünschenswert. 
Konzepte medialer Selbstsozialisation) oder Vereinseitigungen in Richtung einer stark gesellschaftlich-normativen Argumentation. Interessant ist, dass beide Tendenzen milieubezogene Unterschiede zu wenig berücksichtigen.

Konzepte zur Selbstsozialisation mit Medien (u. a. Müller/Rhein/Glogner 2004) betonen die Eigenleistungen der Individuen im Sozialisationsprozess. Diese Eigenleistungen haben im Laufe der letzten zwei Jahrzehnte zweifelsohne zugenommen. Die Bedenken gegenüber dem Konzept medialer Selbstsozialisation beziehen sich nicht auf diesen empirisch belegbaren Prozess, sondern auf die begriffliche Fassung dieses Prozesses sowie auf bestimmte Annahmen, die mit dem Konzept verbunden sind (Niesyto 2007). Eine dieser Annahmen betrifft das Postulat einer starken Autonomie- und Wahlfähigkeit von Individuen, die als "Selbstsozialisierer» ihre Mitgliedschaften in verschiedenen soziokulturellen Kontexten selbst wählen und/oder gestalten: «Wählbar sind neben Gegenständen und Symbolen Mitgliedschaften, Beziehungsformen und Werthaltungen, Deutungsmuster und sinngebende Inhalte, deren Vermittlungsinstanzen die Wahlnachbarschaften sind» (Müller/Rhein /Glogner 2004, S. 240). Müller u. a. weisen darauf hin, dass es eine wichtige Frage ist, "ob und wie individuelle Wahlentscheidungen, die musikalischen und medialen Aktivitäten und Präferenzen zugrunde liegen und sich in Lebensstilmustern niederschlagen, von sozialen Bedingungen wie Geschlecht, Alter, Ethnizität, soziale Schichtzugehörigkeit beeinflusst werden» (Müller u. a. 2002, S. 10). Damit wird generell auch die Bedeutung struktureller, milieubezogener Faktoren angesprochen. Mein Eindruck ist jedoch, dass in der Vergangenheit insbesondere die Erhebung und Analyse unterschiedlicher sozialer und lebenslagenbezogener Bedingungen und Ressourcen zu wenig thematisiert wurde und nicht mit einem entsprechenden Gewicht in Forschungsdesigns Eingang fand. Dies betrifft im Übrigen nicht nur Ansätze zur Selbstsozialisation, sondern einen Grossteil von Jugend- und Medienstudien. Gerade die Überschätzung der Möglichkeiten zu einem medien-autonomen Handeln scheint ein kritischer Punkt in kulturtheoretisch orientierten Kinder- und Jugendmedienstudien zu sein. Dies betrifft insbesondere die Überschätzung der Distanzierungsmöglichkeiten gegenüber problematischen Medienangeboten und medialen Inszenierungsstrategien (vgl. Niesyto 2007). Eher unterschätzt wurde in der Vergangenheit der Einfluss familiärer Sozialisationsbedingungen für jugendliche Mediennutzungsstile sowie die Notwendigkeit milieuspezifischer Ausdifferenzierungen medienpädagogischer Konzepte.

Vereinseitigungen in Richtung einer normativen Verengung des Medienkompetenzbegriffs sind vor allem in Zusammenhang mit gesellschaftlichen Anforderungen und Interessen zu beobachten. Zwar dient Medienkompetenz nicht nur der Allgemeinbildung und Persönlichkeitsbildung, sondern ist gerade heute integraler Bestandteil der Berufsausbildung. Jugendliche, die über zu geringe digitale Medienkompetenzen verfügen, haben grosse Schwierigkeiten, auf dem Arbeitsmarkt Anschluss zu finden, zumal in nahezu allen Branchen digitale Technologien Einzug 
halten. Gleichzeitig ist darauf hinzuweisen, dass Ziele und Anforderungsstrukturen von Medienkompetenz nicht von einem Primat wirtschaftlicher Interessen her definiert werden sollten. Entsprechende Kompetenzprofile sind oft sehr technikorientiert, zielen auf Effektivität und Wettbewerbsfähigkeit und unterschätzen die Bedeutung von (digitalen) Medien für Kommunikation, Orientierung und Identitätsbildung (entsprechende Konzepte kamen in der Vergangenheit vor allem aus der Informatik). Weitere Kritikpunkte an normativen Konzepten sind die einseitige Orientierung auf formale Lernprozesse sowie eine Unterschätzung milieubezogener Förderkonzepte.

Aber auch aus der Medienwissenschaft kommende Konzepte wie z.B. die Wissenskluft-Hypothese sind bezüglich ihrer Implikationen kritisch zu hinterfragen. Die Wissensklufthypothese ist eng mit der Vorstellung verbunden, «dass die politische Informiertheit aller als möglichst homogene Informationsverteilung für das Funktionieren moderner Demokratien [wichtig] ist» (Bonfadelli 2005, S. 10). Was heisst in diesem Zusammenhang «Informiertheit»? Welche Vorstellungen und Qualitätskriterien verbinden sich damit? Es gibt unterschiedliche Formen der «Informiertheit» in der Gesellschaft, so wie es unterschiedliche Wissensarten und Wissensbestände gibt. Bonfadelli und Vollbrecht verweisen in diesem Zusammenhang auf den normativen Aspekt in der Wissenskluftforschung (unkritische Orientierung an Normen der Mittelklasse; vgl. Schäfer/Lojewski 2007, S. 88 f.). Es ist durchaus eine wichtige Frage, wie sich Menschen aus bildungsmässig und sozial benachteiligten Verhältnissen über gesellschaftlich-politische Fragen informieren, sich selbst Meinungen bilden und an gesellschaftlichen Diskursen beteiligen. Da lassen sich durchaus ¿Defizite` feststellen - übrigens nicht nur in Sozialmilieus mit formal niedriger Bildung. Dennoch bleibt die Frage, was die Kriterien für Kompetenz-Definitionen (und damit verknüpfte Zielbestimmungen) sind und wer diese definiert.

Medienkompetenz-Konzepte - so die hier vertretene Auffassung - sollten stärker berücksichtigen, wie die Menschen Medien im konkreten Kontext ihrer Lebenslagen und Lebensbedürfnisse nutzen und welche pragmatischen Medienkompetenzen sie hierfür ausbilden. Entgegen der o.g. Vereinseitigungen geht es um ein Verständnis, das die wechselseitige Verwobenheit von individuellen Handlungsmustern und gesellschaftlich-medialen Angebotsstrukturen im Blick hat und - in handlungstheoretischer Perspektive - die Verarbeitungsleistungen der Subjekte (persönliche, inneres Ressourcen) in Zusammenhang mit den jeweils vorhandenen sozialen Lebenslagen und Anregungsmilieus (als «äussere» Ressourcen) betrachtet.

In dieser Perspektive geht es zunächst einmal um Fragen sozialer Distinktion in Verbindung mit milieu- und lebensstilbezogenen Mustern der Weltaneignung und Lebensbewältigung. Moser verweist in seinen Analysen über Web-2.0-Praktiken auf Arbeiten von Boyd (2007), die in der Nutzung der Onlineportale MySpace und Facebook in den USA Aspekte sozialer Distinktion betont: «Soziale Ungleichheiten 
sind deshalb zu eng gesehen, wenn sie sich allein auf den Faktor ungleich verteilten Wissens beziehen, wie es in der Wissenskluft-Debatte oft erscheint. Kulturelles und soziales Kapital ist jedenfalls nicht zu verwechseln mit der Fähigkeit, sich bestimmtes Wissen anzueignen, sondern es bezieht sich auf Distinktionen wie das Gefühl, welche Leute besser zu einem passen, auf ähnliche Wert- und Präferenzmuster und auf soziale Kontakte - das, was in Bourdieus Worten die ‘feinen Leutes ausmacht. Soziale Netzwerke werden so zu einer Öffentlichkeit, die wichtig für die Sozialisationsprozesse von Jugendlichen werden» (Moser 2008). Soziokulturelle Unterschiede in der Mediennutzung und Mediensozialisation verweisen nicht automatisch auf Aspekte sozialer Benachteiligung und Ungleichheit, sondern zunächst einmal auf andere medien- und sozial-ästhetische Muster und Präferenzen.

Aspekte sozialer Ungleichheit in der Mediennutzung werden vor allem dann sichtbar, wenn es um vorhandene (innere und äussere) Ressourcen geht, um z. B. kommunikationskulturelle Problemlagen zu bewältigen. Baacke benutzte den Begriff «kommunikationskulturelle Problemlagen», um insbesondere auf folgende Problemfelder hinzuweisen: Orientierungsdilemma zwischen Medien und Arbeitswelt; Schwierigkeit des Unterscheidens; Optionenvielfalt und Schwierigkeit, sich zu entscheiden; Ver-Oberflächlichung von Wahrnehmungstätigkeiten (Baacke 1997, S. $76 f f$.$) . Sich für neue Wahrnehmungsformen zu öffnen, ist das eine; sich reflexiv$ mit Fragen der Optionenvielfalt, Strategien medialer Aufmerksamkeitserregung, der Ver-Oberflächlichung von Wahrnehmung und des Umgangs mit persönlichen Daten auseinanderzusetzen, ist das andere. Wenngleich Studien reflexive, an Wertkategorien orientierte Wahl- und Urteilsprozesse konstatieren (z. B. Marci-Boehncke/Rath 2006), so ist doch davon auszugehen, dass der von Medienangeboten ausgehende Konsum- und Konformitätsdruck und die oft fehlenden familiären Anregungen vielen Kindern und Jugendlichen nur eingeschränkte Möglichkeiten lassen. Das Bild vom «autonomen Rezipienten`, der Zugriff auf sehr viele mediale Ressourcen hat und in freier Entscheidung daraus gezielt auswählt, mag für bestimmte Gruppen von Kindern und Jugendlichen zutreffen. Es gibt aber sehr viele Kinder und Jugendliche, die weder im Rahmen der familiären noch der schulischen Sozialisation hinreichend Anregung und Förderung für einen reflektierten Medienumgang erhalten. Entsprechende Sozialisationskonstellationen verweisen auf ein komplexes Problemfeld, das u. a. der 12. Kinder- und Jugendbericht der Bundesregierung analysierte. Dort heisst es in einem Abschnitt über Medien als Verstärker von sozialer Ungleichheit, der auf eine Expertise von Helga Theunert (2005) zurückgeht: «Auch wenn der Umgang mit Medien als Bildungsprozess mit zunehmendem Alter zu einer selbständigen Angelegenheit der Jugendlichen wird, so basiert die Verarbeitung der Medienerlebnisse und Medieninformationen auf den sozio-kulturellen Voraussetzungen der jeweiligen Familie. Es geht demnach nicht nur um die Frage der Zugänge zu Medien, sondern verstärkt auch um die Frage danach, wer sich welche Medienangebote auf welche Weise und aus 
welcher Motivlage heraus aneignet bzw. welche Folgen dies für die individuelle Entwicklung und für die gesellschaftliche Partizipation hat. Ist der Medienumgang einer selbst bestimmten und souveränen Lebensführung zuträglich, dann vermehrt die Nutzung von (konvergenten) Medienangeboten die wünschenswerten Effekte informellen Lernens mit Medien, was jedoch mehr für bildungsbevorzugte als für bildungsbenachteiligte Jugendliche zutrifft. Andererseits kann ein konvergierendes Medienensemble bei einem problematischen Medienumgang die negativen Effekte verstärken» (Bundesministerium für Familie, Senioren, Frauen und Jugend 2005, S. 241).

In dieser Situation ist es erforderlich, medienpädagogische Beratungs- und Förderangebote auszubauen. Notwendig ist die Entwicklung von Förderangeboten, die an den alltäglichen Medienerfahrungen der Jugendlichen ansetzen und ihnen - in formellen und non-formalen Bildungskontexten - Chancen eröffnen, vorhandene Medienkompetenzen zu vertiefen. Im Bereich der schulischen Medienbildung besteht hier das Problem einer Kluft zwischen den medienbezogenen Grundhaltungen bei vielen Pädagogen/-innen einerseits und vielen Schülern andererseits (Kommer/Biermann 2007; Kommer 2006). Diese Kluft wird insbesondere dann zu einem gravierenden Problem, wenn eine oberflächliche Medienkritik mit Abwertungen von Medienpräferenzen bei Kindern und Jugendlichen und mit fehlenden Medienkompetenzen bei Lehrkräften verbunden ist. Anstatt kognitionslastige Konzepte zu favorisieren, die Sinnlichkeit, Intuition und Assoziation in der Wahrnehmung unterschätzen, sollten Pädagogen/-innen genau hinschauen, wie Kinder und Jugendliche aus benachteiligten Sozialmilieus mit traditionellen und neuen Medien umgehen, welche Formen des Zugangs, der Aneignung und des Ausdrucks sie bevorzugen und entwickeln. Was wir brauchen, sind zielgruppenspezifische Konzepte, die ästhetisch, sozial und kommunikativ auf die jeweiligen Bedürfnisse, Aneignungsformen und Themen hin ausgelegt sind (vgl. Niesyto 2000; Maurer 2004; Hoffmann 2006).

Nach wie vor sind viele Pädagogen/-innen noch zu sehr in einer bildungsbürgerlichen Symbolsozialisation befangen, die auf dem Diskursiven, auf dem Wort- und Schriftsprachlichen beruht. Sie haben oft Angst, sich auf bestimmte Gesten, körperliche Ausdrucksformen, symbolische Codes einzulassen. Die Förderung der Medienkompetenz von Kindern und Jugendlichen aus benachteiligten Sozialmilieus setzt qualifizierte Pädagogen/-innen voraus, die über eigene Medienkompetenzen, über ein Wissen zum Sozialisationshintergrund von Kindern und Jugendlichen sowie über geeignete gruppenpädagogische und methodisch-didaktische Qualifikationen verfügen. Hierfür ist es notwendig, medienpädagogische Inhalte in der Ausbildung von pädagogischen Fachkräften verbindlich in Studien- und Prüfungsordnungen zu verankern (Niesyto 2008). Es geht dabei nicht nur um Wissensbestände, sondern auch um die Reflexion eigener Haltungen gegenüber Medien. Die Entwicklung von differenzierter Medienkritik ist ohne medienbiogra- 
fische Selbstreflexionen der Studierenden nicht möglich. Diese Selbstreflexionen sind eine Voraussetzung für ein zielgruppenbezogenes Handeln, das alters-, bildungs-, geschlechts- und milieubezogene Unterschiede berücksichtigt und (implizite und explizite) normative Orientierungen in medienpädagogischen Konzepten hinterfragt. Das Problem einer «Mittelschichtlastigkeit) (medien)pädagogischer Konzepte (Niesyto 2008) ist im Übrigen nicht nur an Schulen vorhanden. Es ist mitunter auch ein Problem im ausserschulischen Bereich wie z. B. eine Studie von Welling zu den Computerpraxen von Jugendlichen und den Formen medienpädagogischen Handelns bei Jugendhaus-Mitarbeitern/-innen zeigt. Welling analysiert im Rahmen von Fallstudien, dass die Wahrnehmung der Computerpraxen durch die Jugendarbeiter/innen «über weite Strecken von defizitären Zuschreibungen im Sinne eingeschränkter Kenntnisse und Interessen» geprägt sei (Welling 2008, S. 211). Nach Einschätzung der Jugendarbeiter/innen würden die Jugendlichen nur über geringe Kenntnisse im Umgang mit digitalen Medien verfügen und sich auf wenige, relativ anspruchslose Praxen wie z. B. Chatten beschränken. Gegenüber solchen defizitären Zuschreibungen plädiert Welling dafür, die Medienpraxis Jugendlicher auf dem Hintergrund milieuspezifischer Orientierungsmuster und biografischer Relevanzen zu rekonstruieren und zu verstehen. Nur auf dieser Basis können lebensweltliche Gestaltungsperspektiven im Sinne einer (medien-)pädagogischen Milieubildung entwickelt werden (ebd., S. 265).

\section{Literatur}

Baacke, Dieter (1997). Medienpädagogik. Tübingen: Niemeyer.

Bachmair, Ben (2009). Medienwissen für Pädagogen. Medienbildung in riskanten Erlebniswelten. Wiesbaden: VS Verlag.

Bonfadelli, Heinz (2005). Die Rolle digital-interaktiver Medien für gesellschaftliche Teilhabe. In: medien + erziehung, 49. Jhg., Nr. 6, S. 6-16.

Bourdieu, Pierre (1970). Zur Soziologie der symbolischen Formen. Frankfurt/Main: Suhrkamp.

Bourdieu, Pierre (1979 ${ }^{1972}$ ). Entwurf einer Theorie der Praxis auf der Grundlage der kabylischen Gesellschaft. Frankfurt/Main: suhrkamp.

Bourdieu, Pierre (1987 ${ }^{1979}$ ). Die feinen Unterschiede. Kritik der gesellschaftlichen Urteilskraft. Frankfurt/Main: suhrkamp.

Boyd, Danah (2007). «Why Youth (Heart) Social Network Sites: The Role of Networked Publics in Teenage Social Life.» MacArthur Foundation Series on Digital Learning - Youth, Identity, and Digital Media Volume (ed. David Buckingham). Cambridge, MA: MIT Press, pp. 119-142. [pdf] URL: www.danah.org/papers/WhyYouthHeart.pdf [01.04.09]

Bundesregierung (Hg.) (2008). Lebenslagen in Deutschland. Der 3. Armuts- und Reichtumsbericht der Bundesregierung. URL: http://www.bmas.de/coremedia/generator/26892/ property=pdf/dritter__armuts__und_reichtumsbericht_kurzfassung.pdf [01.04.09]

Bundesministerium für Familie, Senioren, Frauen und Jugend (Hg.) (2002). Elfter Kinder- und Jugendbericht der Bundesergierung. URL: www.dji.de/bibs/Elfter_Kinder_und_Jugendbericht.pdf [01.04.09] 
Charlton, Michael (1997). Rezeptionsforschung als Aufgabe einer interdisziplinären Medienwissenschaft. In: Charlton, Michael; Schneider, Sylvia (Hg.). Rezeptionsforschung. Opladen: Westdeutscher Verlag, S. 16-39.

Charlton, Michael; Neumann, Klaus (1986). Medienkonsum und Lebensbewältigung in der Familie. Methode und Ergebnisse der strukturanalytischen Rezeptionsforschung - mit fünf Falldarstellungen. München/Weinheim: Psychologie Verlags Union.

Chinn, Menzie; Fairlie, Robert (2006). The determinants of the global digital divide: a crosscountry analysis of computer and internet penetration. Oxford Economic Papers.

Eimeren, Birgit van; Frees, Beate (2008). Internet-Verbreitung: Grösster Zuwachs bei SilverSurfern. In: Media Perspektiven, Nr. 7, S. 330-344.

Eisemann, Christoph (2008). «Why do they tube? Aspekte zur Nutzung von Videoplattformen durch Jugendliche und junge Erwachsene.» In: Onlinemagazin Ludwigsburger Beiträge zur Medienpädagogik, Ausgabe 12/2008. URL: www.ph-ludwigsburg.de/fileadmin/subsites/1b-mpxx-t-01/user_files/Online-Magazin/Ausgabe11/Forschung11.pdf [01.04.09]

Empirica Gesellschaft für Kommunikations- und Technologieforschung mbH (Bonn) / Work Research Centre (Dublin) (2007). MeAC - Measuring Progress of eAccessibility in Europe. URL: www.eaccessibility-progress.eu/wp-content/uploads/2008/04/meac_report_06_11_final.pdf

Gerhards, Maria; Mende, Annette (2008). Ein Drittel der Deutschen bleibt weiter offline. In: Media Perspektiven Nr. 7, S. 365-376.

Gerhards, Maria; Mende, Annette (2007). Offliner 2007: Zunehmend distanzierter, aber gelassener Blick aufs Internet. In: Media Perspektiven Nr. 8, S. 379-392.

Groeben, Norbert; Hurrelmann, Bettina (Hg.) (2002). Lesekompetenz: Bedingungen, Dimensionen, Funktionen. Weinheim und München: Juventa.

Hargittai, Eszter (2007). Characteristics of use differences and their implications for dealing with digital inequality. In: Kompetenzzentrum Informelle Bildung (Hg.). Grenzenlose Cyberwelt? Zum Verhältnis von digitaler Ungleichheit und neuen Bildungszugängen für Jugendliche. Wiesbaden: VS-Verlag, S. 121-136.

Hoffmann, Bernward (2006). Medienkompetenz sozial benachteiligter Kinder. In: tv diskurs, 10. Jhg., Nr. 4/06, 14-17.

Holloway, Sarah L.; Valentine, Gil (2003). Cyberkids. Children in the Information Age. London: Rotledge/Falmer.

Holzwarth, Peter (2008). Migration, Medien und Schule. Fotografie und Video als Zugang zu Lebenswelten von Kindern und Jugendlichen mit Migrationshintergrund. Reihe Medienpädagogische Praxisforschung, Bd. 3. München: kopaed.

Hradil, Stefan (2001). Soziale Ungleichheit in Deutschland. Opladen: Leske + Budrich.

Hurrelmann, Klaus (1995). Einführung in die Sozialisationstheorie. Über den Zusammenhang von Sozialstruktur und Persönlichkeit. Weinheim/Basel: Beltz.

Initi@tive D 21 (2008). (N)Onliner Atlas 2008. Eine Topographie des digitalen Grabens durch Deutschland. URL: http://old.initiatived21.de/fileadmin/files/08_NOA/NONLINER2008. pdf

Iske, Stefan; Klein, Alexandra; Kutscher, Nadia; Otto, Hans-Uwe (2007). Virtuelle Ungleichheit und informelle Bildung. Eine empirische Analyse der Internetnutzung Jugendlicher und ihre Bedeutung für Bildung und gesellschaftliche Teilhabe. In: Kompetenzzentrum Informelle Bildung (Hg.). Grenzenlose Cyberwelt? Zum Verhältnis von digitaler Ungleichheit und neuen Bildungszugängen für Jugendliche. Wiesbaden: VS-Verlag, S. 65-91. 
Kommer, Sven; Biermann, Ralf (2007). Zwischen Erinnerung und Inszenierung - Medienbiografien medial. In: Freiburger FrauenStudien «Erinnern und Geschlecht, Band II», Band 20, S. 195-220.

Kommer, Sven (2006). Zum medialen Habitus von Lehramtsstudierenden. Oder: Warum der Medieneinsatz in der Schule eine so «schwere Geburt) ist. In: Treibel, Annette/Maier, Maja S./Kommer, Sven/Welzel, Manuela (Hg.). Gender medienkompetent. Medienbildung in einer heterogenen Gesellschaft. Wiesbaden: VS-Verlag, S. 165-177.

Krais, Beate; Gebauer, Gunter (2002). Habitus. Bielefeld: transcript.

Livingstone, Sonja; Bober, Magdalena (2004). UK Children Go Oneline. Surveying the Experiences of Young People and their Parents, London : Economic \& Social Research Council.

Marci-Boehncke, Gudrun; Rath, Matthias (Hg.) (2006). Jugend - Werte - Medien: Der Diskurs. Weinheim: Beltz.

Maurer, Björn (2004). Medienarbeit mit Kindern aus Migrationskontexten. Grundlagen und Praxisbausteine. Reihe Medienpädagogische Praxisforschung, Bd. 1. München: kopaed.

Medienpädagogischer Forschungsverbund Südwest (Hg.) (2008). JIM-Studie 2008 Jugend, Information (Multi-) Media. Stuttgart: Landesanstalt für Kommunikation.

Medienpädagogischer Forschungsverbund Südwest (Hg.) (2005). JIM-Studie 2005 Jugend, Information (Multi-) Media. Stuttgart: Landesanstalt für Kommunikation.

Mikos, Lothar (2007). Distinktionsgewinne - Diskurse mit und übe Medien. In: Fromme, Johannes; Schäffer, Burkhard (Hg.). Medien - Macht - Gesellschaft. Wiesbaden: VS-Verlag, S. 45-60.

Moser, Heinz (2008). Medienkritik in den Zeiten von Digital Divide und Web 2.0. Vortrag in $\operatorname{der}$ AG 47 auf dem 21. DGfE-Kongress in Dresden. Unveröffentlichtes Manuskript.

Moser, Heinz (2006). Medien und die Konstruktion von Identität und Differenz. In: Treibel, Annette; Maier, Maja S.; Kommer, Sven; Welzel, Manuela (Hg.). Gender medienkompetent. Medienbildung in einer heterogenen Gesellschaft. Wiesbaden: VS-Verlag, S. 53-74.

Müller, Renate; Rhein, Stefanie; Glogner, Patrick (2004). Das Konzept musikalischer und medialer Selbstsozialisation - widersprüchlich, trivial, überflüssig? In: Hoffmann, Dagmar; Merkens, Hans (Hrsg.). Jugendsoziologische Sozialisationstheorie. Impulse für die Jugendforschung. Weinheim und München: Juventa, S. 237-252.

Müller, Renate; Glogner, Patrick; Rhein, Stefanie; Heim, Jens (Hg.) (2002). Wozu Jugendliche Musik und Medien gebrauchen. Jugendliche Identität und musikalische und mediale Geschmacksbildung. Weinheim und München: Juventa.

Niesyto, Horst (2008). Wohin wird die Reise gehen? Anforderungen einer zukunftsorientierten Schule an eine nachhaltige Medienbildung als Teil der Lehrerausbildung von heute. In: Onlinemagazin Ludwigsburger Beiträge zur Medienpädagogik, Ausgabe 11/2008. URL: www.ph-ludwigsburg.de/fileadmin/subsites/1b-mpxx-t-01/user_files/Niesyto_nachhaltige_Medienbildung.pdf [01.04.09]

Niesyto, Horst (2007). Kritik zu phänomenologisch und kulturalistisch verkürzten Auffassungen in Jugend- und Mediensozialisationstheorien. In: Göttlich, Udo; Müller, Renate; Calmbach, Marc (Hg.). Arbeit, Politik und Religion in Jugendkulturen. Engagement und Vergnügen. Weinheim und München: Juventa, S. 41-55.

Niesyto, Horst (Hg.) (2003). VideoCulture - Video und interkulturelle Kommunikation. München: kopaed. 
Niesyto, Horst (2002). Medien und Wirklichkeitserfahrung - symbolische Formen und soziale Welt. In: Mikos, Lothar; Neumann, Norbert (Hg.). Wechselbeziehungen. Medien - Wirklichkeit - Erfahrung. Berlin: Vistas, S. 29-54.

Niesyto, Horst (2000). Medienpädagogik und soziokulturelle Unterschiede. Studie im Auftrag des Medienpädagogischen Forschungsverbunds Südwest. Baden-Baden/Ludwigsburg.

Paus-Hasebrink, Ingrid (2006). Medienpädagogische Forschung braucht gesellschaftskritischen Handlungsbezug. Besondere Verantwortung gebührt sozial benachteiligten Kindern und Jugendlichen. In: medien + erziehung, 50. Jhg., Nr. 5, S. 22-28.

PISA-Konsortium Deutschland (Hg.) (2004). PISA 2003. Der Bildungsstand der Jugendlichen in Deutschland - Ergebnisse des zweiten internationalen Vergleichs. Münster.

Rainie, Lee (2008). Increased Use of Video-sharing Sites. Data Memo. PEW Internet \& American Life Project. Supported by MacArthur Foundation. URL: www.pewinternet.org/Reports/2008/Increased-Use-of-Videosharing-Sites.aspx [01.04.09]

Rehberg, Karl-Siegbert (Hg.) (2006). Soziale Ungleichheit, Kulturelle Unterschiede, Verhandlungen des 32. Kongresses der Deutschen Gesellschaft für Soziologie in München 2004, 2 Bände mit CD-ROM. Frankfurt / New York: Campus.

Schäfer, Miriam; Lojewski, Johanna (2007). Internet und Bildungschancen. Die soziale Realität des virtuellen Raumes. München: kopaed.

Schorb, Bernd; Keilhauer, Jan; Würfel, Maren; Kiessling, Matthias unter Mitarbeit von Michael Baumann (2009). Medienkonvergenz Monitoring Report 2008. Jugendliche in konvergenten Medienwelten. Im Internet unter: http://www.uni-leipzig.de/ umfmed/Medienkonvergenz_Monitoring_Report08.pdf [01.04.09]

Schwinn, Thomas (2006). Ungleichheitsstrukturen versus Vielfalt der Lebensführungen. In: Rehberg, Karl-Siegbert (Hrsg.). Soziale Ungleichheit, Kulturelle Unterschiede, Verhandlungen des 32. Kongresses der Deutschen Gesellschaft für Soziologie in München 2004, Band 2, Frankfurt / New York, Campus: S. 1283-1297.

Theunert, Helga (2005). Medien als Orte informellen Lernens im Prozess des Heranwachsens. Expertise zum Zwölften Kinder- und Jugendbericht, hgg. Vom Bundesministerium für Familie, Senioren, Frauen und Jugend, Berlin. URL: www.bmfsfj.de/RedaktionBMFSFJ/Abteilung5/Pdf-Anlagen/zwoelfter-kjb, property=pdf.pdf

Treibel, Annette (2004). Einführung in soziologische Theorien der Gegenwart. 6. Aufl. Wiesbaden: VS-Verlag.

Treumann, Klaus Peter; Meister, Dorothee M.; Sander, Uwe; Burkatzki, Eckhard; Hagedorn, Jörg; Kämmerer, Manuela; Strotmann, Mareike; Wegener, Claudia (Hg.) (2007). Medienhandeln Jugendlicher. Mediennutzung und Medienkompetenz. Bielefelder Medienkompetenzmodell. Wiesbaden: VS-Verlag.

Wagner, Ulrike; Eggert, Susanne (2007). Quelle für Information und Wissen oder unterhaltsame Action? Bildungsbenachteiligung und die Auswirkungen auf den Medienumgang Heranwachsender. In: medien + erziehung, 51. Jahrgang, Nr. 5, S. 15-23.

Wagner, Ulrike (Hg.) (2008). Medienhandeln in Hauptschulmilieus. Mediale Interaktion und Produktion als Bildungsressource. München: kopaed.

Welling, Stefan (2008). Computerpraxis Jugendlicher und medienpädagogisches Handeln. Reihe Medienpädagogische Praxisforschung, Bd. 4. München: kopaed.

Witzke, Margrit (2004). Identität, Selbstausdruck und Jugendkultur. Eigenproduzierte Videos Jugendlicher im Vergleich mit ihren Selbstaussagen. Ein Beitrag zur Jugend(kultur) forschung. München: kopaed. 